\title{
Editorial
}

\section{Metals in 3D: A Cosmic View from Integral Field Spectroscopy}

\author{
Jorge Iglesias-Páramo \\ Instituto de Astrofísica de Andalucía, CSIC, 18008 Granada, Spain \\ Correspondence should be addressed to Jorge Iglesias-Páramo; jiglesia@iaa.es
}

Received 8 April 2014; Accepted 8 April 2014; Published 21 May 2014

Copyright (c) 2014 Jorge Iglesias-Páramo. This is an open access article distributed under the Creative Commons Attribution License, which permits unrestricted use, distribution, and reproduction in any medium, provided the original work is properly cited.

Integral field spectroscopy (IFS) is an observational technique that has revolutionized our understanding of extended objects in the Universe since the last decade. IFS provides unique contributions to the study of Galactic ionized nebulae, extragalactic HII regions, and galaxies in the local and in the early Universe, which in some aspects are complementary to the high precision performance of existing wide area photometric and single-aperture spectroscopic surveys. The power of this technique is the possibility of gathering complete spectroscopic information across the spatial extension of astrophysical objects, thus allowing the study of physical spatial variations of their properties, this being specially significant for those properties related to their metal content and star formation activity. With this special issue we want to cover the most relevant aspects related to IFS, in order to illustrate to the reader the wide scope opened by this observational technique.

In what follows we present the main contents of this special issue split by subject. Two papers of this issue are devoted to modeling the chemical evolution of spiral and dwarf galaxies by M. Mollá and S. Recchi. Theoretical models are a key ingredient in order to be compared with the observational data since either their predictions open new observational avenues or they are constrained by new observational results. Both aspects must walk hand in hand to make progress in the field.

Two papers follow, dealing with the study of Galactic objects. The one by L. López-Martín mostly addresses some aspects of the particular technical problems of IFS data handling. A paper by A. Mesa-Delgado faces the chemical abundance discrepancy problem applied to Galactic Herbig Haro objects in the Orion nebula, illustrating how IFS can help to shed light on this issue.
A set of four papers follows focused on extragalactic studies with fiber-fed spectrographs: the first two papers, by E. Pérez-Montero et al. and P. Lagos and P. Papaderos, deal with the chemical properties of star-forming (HII/BCD) dwarf galaxies and with the interplay of the WR stellar population on the metal enrichment of the interstellar medium. The remaining two papers of this block, by F. F. RosalesOrtega and S. F. Sánchez, are focused on the properties of the HII regions in spiral galaxies and review most recent results on chemical abundance gradients obtained from IFS observations.

Last but not least, a set of two papers of this issue are devoted to the description and main properties of two ongoing experiments/instruments. One is a wide-field integral field spectrograph, by G. A. Blanc, with applications in several astrophysical fields, in particular in the study of the properties of nearby galaxies. The second one, by L. Drissen et al., explains the basics of the operation of imaging Fourier transform spectrometers (IFTS), an interesting alternative to the traditional dispersive spectrographs due to their wide field of view, and it shows the main goals that can be achieved using them in the study of the chemical evolution of astrophysical objects.

Jorge Iglesias-Páramo 

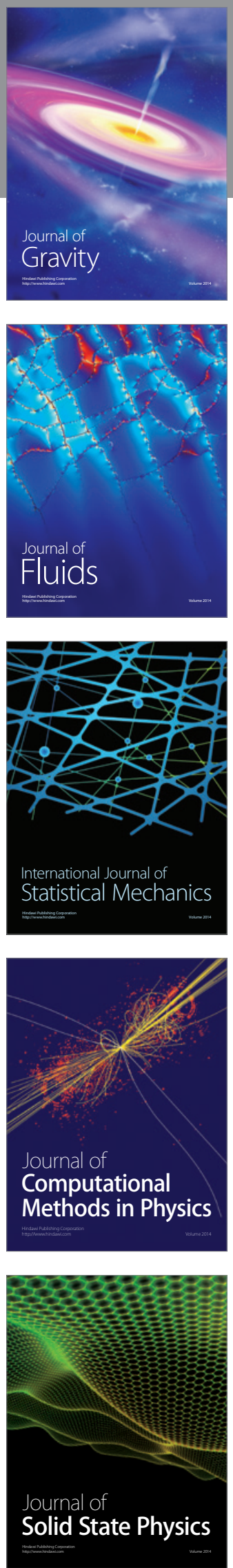

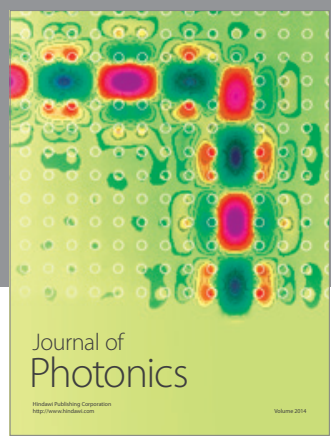

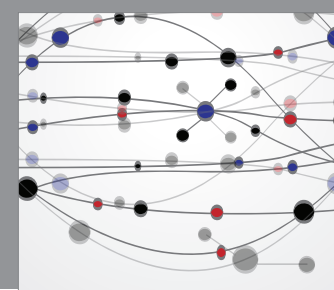

The Scientific World Journal

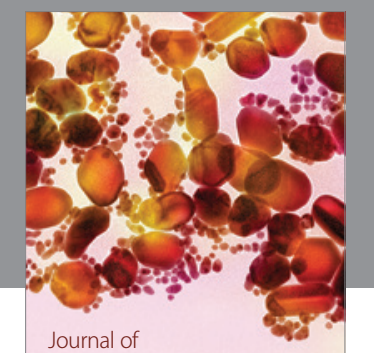

Soft Matter
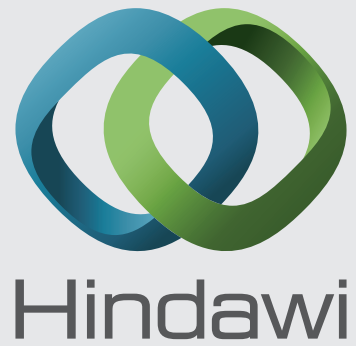

Submit your manuscripts at

http://www.hindawi.com
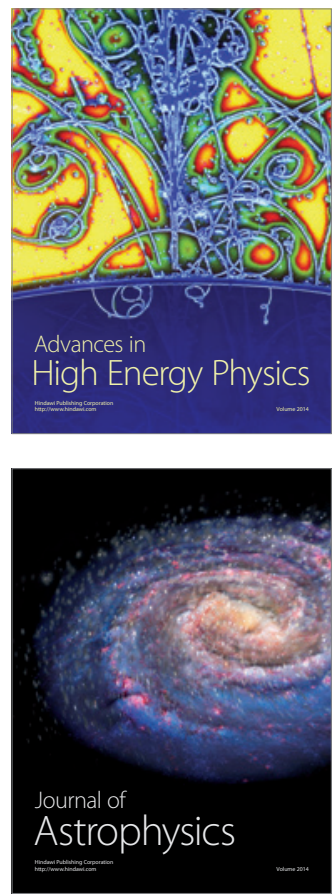
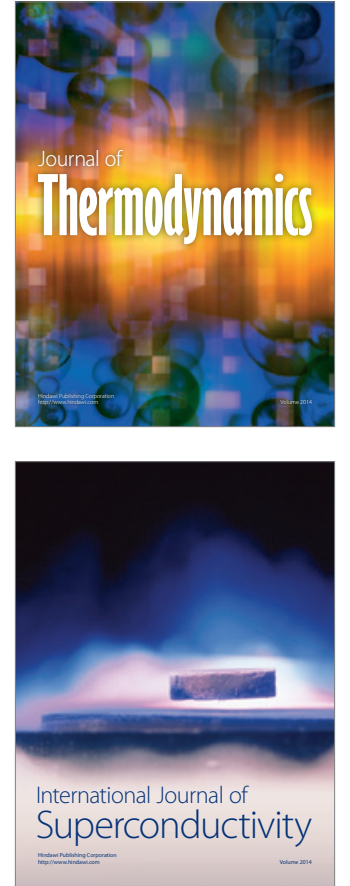
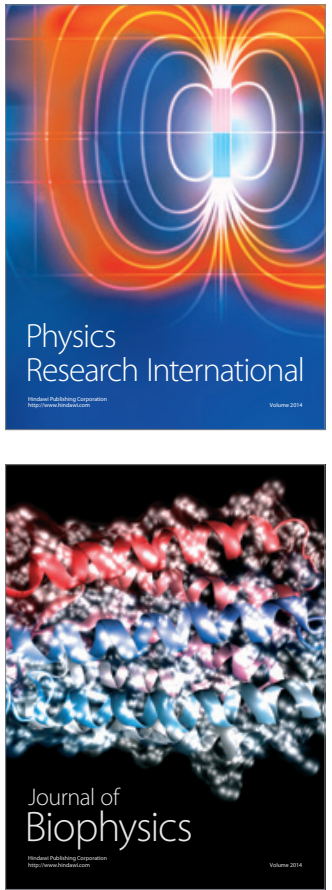
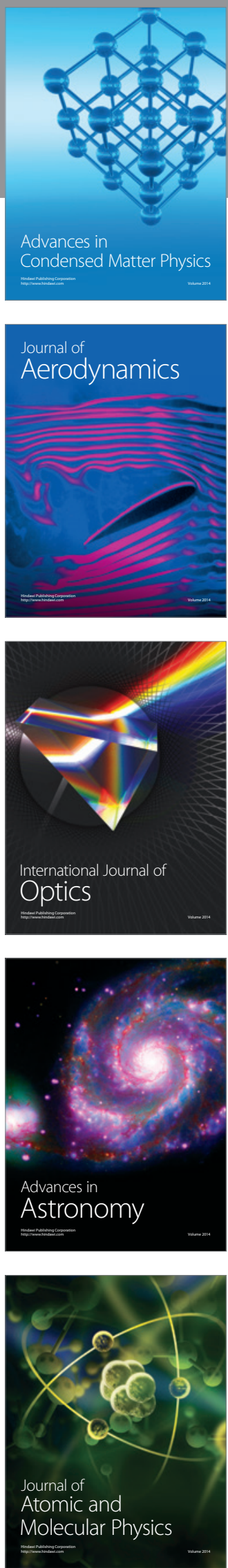